\title{
UAlacant machine translation quality estimation at WMT 2018: a simple approach using phrase tables and feed-forward neural networks
}

\author{
Miquel Esplà-Gomis Felipe Sánchez-Martínez Mikel L. Forcada \\ Departament de Llenguatges i Sistemes Informàtics \\ Universitat d'Alacant, E-03071 Alacant, Spain \\ \{mespla, fsanchez,mlf\}edlsi.ua.es
}

\begin{abstract}
We describe the Universitat d'Alacant submissions to the word- and sentence-level machine translation (MT) quality estimation (QE) shared task at WMT 2018. Our approach to word-level MT QE builds on previous work to mark the words in the machine-translated sentence as $O K$ or $B A D$, and is extended to determine if a word or sequence of words need to be inserted in the gap after each word. Our sentence-level submission simply uses the edit operations predicted by the word-level approach to approximate TER. The method presented ranked first in the sub-task of identifying insertions in gaps for three out of the six datasets, and second in the rest of them.
\end{abstract}

\section{Introduction}

This paper describes the Universitat d'Alacant submissions to the word- and sentence-level machine translation (MT) quality estimation (QE) shared task at WMT 2018 (Specia et al., 2018). Our approach is an extension of a previous approach (Esplà-Gomis et al., 2015a,b; Esplà-Gomis et al., 2016) in which we simply marked the words $t_{j}$ of a machine-translated segment $T$ as $O K$ (no changes are needed) or as $B A D$ (needing editing). Now we also mark the gaps $\gamma_{j}$ after each word $t_{j}$ as $O K$ (no insertions are needed) or as $B A D$ (needing the insertion of one or more words). In addition, we use the edit operations predicted at the word level to estimate quality at the sentence level.

The paper is organized as follows: section 2 briefly reviews previous work on word-level MT QE; section 3 describes the method used to label words and gaps, paying special attention to the features extracted (sections 3.1 and 3.2) and the neural network (NN) architecture and its training (section 3.3); section 4 describes the datasets used; section 5 shows the main results; and, finally, section 6 closes the paper with concluding remarks.

\section{Related work}

Pioneering work on word-level MT QE dealt with predictive/interactive MT (Gandrabur and Foster, 2003; Blatz et al., 2004; Ueffing and Ney, 2005, 2007), often under the name of confidence estimation. Estimations relied on the internals of the actual MT system - for instance, studying the $n$ best translations (Ueffing and Ney, 2007) — or used external sources of bilingual information; for instance, both Blatz et al. (2004) and Ueffing and Ney (2005) used probabilistic dictionaries; in the case of Blatz et al. (2004), as one of many features in a binary classifier for each word.

The last decade has witnessed an explosion of work in word-level MT QE, with most of the recent advances made by participants in the shared tasks on MT QE at the different editions of the Conference on Statistical Machine Translation (WMT). Therefore, we briefly review those papers related to our approach: those using an external bilingual source such as an MT system and those using NN.

As regards work using external bilingual resources, we can highlight four groups of contributions:

- To estimate the sentence-level quality of MT output for a source segment $S$, Biçici (2013) chooses sentence pairs from a parallel corpus which are close to $S$, and builds an SMT system whose internals when translating $S$ are examined to extract features.

- MULTILIZER, one of the participants in the sentence-level MT QE task at WMT 2014 (Bojar et al., 2014) uses other MT systems to translate $S$ into the target language (TL) and $T$ into the source language (SL). The results are compared to the original SL and TL segments to obtain indicators of quality.

- Blain et al. (2017) use bilexical embeddings (obtained from SL and TL word embeddings 
and word-aligned parallel corpora) to model the strength of the relationship between SL and TL words, in order to estimate sentencelevel and word-level MT quality.

- Finally, Esplà-Gomis et al. (2015a,b), and Esplà-Gomis et al. (2016) perform word-level MT QE by using other MT systems to translate sub-segments of $S$ and $T$ and extracting features describing the way in which these translated sub-segments match sub-segments of $T$. This is the work most related to the one presented in this paper.

Only the last two groups of work actually tackle the problem of word-level MT QE, and none of them are able to identify the gaps where insertions are needed.

As regards the use of neural networks (NN) in MT QE, we can highlight a few contributions:

- Kreutzer et al. (2015) use a deep feed-forward $\mathrm{NN}$ to process the concatenated vector embeddings of neighbouring TL words and (wordaligned) SL words into feature vectors extended with the baseline features provided by WMT15 (Bojar et al., 2015) organizersto perform word-level MT QE.

- Martins et al. (2016) achieved the best results in the word-level MT QE shared task at WMT 2016 (Bojar et al., 2016) by combining a feedforward $\mathrm{NN}$ with two recurrent NNs whose predictions were fed into a linear sequential model together with the baseline features provided by the organizers of the task. An extension (Martins et al., 2017) uses the output of an automatic post-editing tool, with a clear improvement in performance.

- Kim et al. (2017a,b) obtained in WMT 2017 (Bojar et al., 2017) results which were better or comparable to those by Martins et al. (2017), using a three-level stacked architecture trained in a multi-task fashion, combining a neural word prediction model trained on large-scale parallel corpora, and word- and sentence-level MT QE models.

Our approach uses a much simpler architecture than the last two approaches, containing no recurrent NNs, but just feed-forward NNs applied to a fixedlength context window around the word or gap about which a decision is being made (similarly to a convolutional approach). This makes our approach easier to train and parallelize.

\section{Method}

The approach presented here builds on previous work by the same authors (Esplà-Gomis et al., 2015a,b; Esplà-Gomis et al., 2016) in which insertion positions were not yet predicted and a slightly different feature set was used. As in the original papers, here we use black-box bilingual resources from the Internet. In particular, we use, for each language pair, the statistical MT phrase tables available at OPUS ${ }^{1}$ to spot sub-segment correspondences between the SL segment $S$ and its machine translation $T$ into the TL (see section 4.2 for details). This is done by dividing both $S$ and $T$ into all possible (overlapping) sub-segments, or $n$-grams, up to a certain maximum length. ${ }^{2}$ These sub-segments are then translated into the TL and the SL, respectively, by means of the phrase tables mentioned (lowercasing of sub-segments before and after translation is used to increase the chance of a match). These sub-segment correspondences are then used to extract several sets of features that are fed to a feed-forward NN in order to label the words and the gaps between words as $O K$ or as $B A D$. One of the main advantages of this approach, when compared to the other approaches described below, is that it uses simple string-level bilingual information extracted from a publicly available source to build features that allow us to easily estimate quality for the words and inter-word gaps in $T$.

\subsection{Features for word deletions}

We define three sets of features to detect the words to be deleted: one taking advantage of the subsegments $\tau$ that appear in $T, \operatorname{Keep}_{n}(\cdot)$; another one that uses the translation frequency with which a sub-segment $\sigma$ in $S$ is translated as the sub-segment $\tau$ in $T$, Freq ${ }_{n}^{\text {keep }}(\cdot)$; and a third one that uses the alignment information between $T$ and $\tau$ and which does not require $\tau$ to appear as a contiguous subsegment in $T, \operatorname{Align}_{n}^{\text {keep }}(\cdot)$.

Features for word deletions based on subsegment pair occurrences (Keep) Given a set of sub-segment pairs $M=\{(\sigma, \tau)\}$ coming from the union of several phrase tables, the first set of features, $\operatorname{Keep}_{n}(\cdot)$, is obtained by computing the amount of sub-segment translations $(\sigma, \tau) \in M$ with $|\tau|=n$ that confirm that word $t_{j}$ in $T$ should be kept in the translation of $S$. A sub-segment

\footnotetext{
${ }^{1}$ http: // opus.nlpl.eu/

${ }^{2}$ For our submission, we used $L=5$.
} 
translation $(\sigma, \tau)$ confirms $t_{j}$ if $\sigma$ is a sub-segment of $S$, and $\tau$ is an $n$-word sub-segment of $T$ that covers position $j$. This set of features is defined as follows:

$$
=\frac{\mid\left\{\tau:(\sigma, \tau) \in \operatorname{Konf}_{n} \operatorname{Keep}_{n}(j, S, T, M)=\right.}{\mid\{\tau: \tau, T, M)\} \mid}
$$

where $\operatorname{seg}_{n}(X)$ represents the set of all possible $n$-word sub-segments of segment $X$, and function $\operatorname{span}(\tau, T)$ returns the set of word positions spanned by the sub-segment $\tau$ in the segment $T$; if $\tau$ is found more than once in $T$, it returns all the possible positions spanned. Function $\operatorname{conf}_{n}^{\text {keep }}(j, S, T, M)$ returns the collection of subsegment pairs $(\sigma, \tau)$ that confirm a given word $t_{j}$, and is defined as:

$$
\begin{array}{r}
\operatorname{conf}_{n}^{\text {keep }}(j, S, T, M)= \\
=\left\{(\sigma, \tau) \in \operatorname{match}_{n}(M, S, T): j \in \operatorname{span}(\tau, T)\right\}
\end{array}
$$

where $\left.\operatorname{match}_{n}(M, S, T)\right)$ is the set of phrase pairs in $M$ with $n$ words in the target that are found in the segment pair $(S, T)$, and where $\operatorname{seg}_{*}(X)$ is similar to $\operatorname{seg}_{n}(X)$ but without length constraints. ${ }^{3}$

Features for word deletions based on subsegment pair occurrences using translation frequency $\left(\right.$ Freq $_{n}^{\text {keep}}$ ) The second set of features uses the probabilities of subsegment pairs. To obtain these probabilities from a set of phrase tables, we first use the count of joint occurrences of $(\sigma, \tau)$ provided in each phrase table. Then, when looking up a SL sub-segment $\sigma$, the probability $p(\tau \mid \sigma)$ is computed across all phrase tables from the accumulated counts. Finally, we define $\operatorname{Freq}_{n}^{\mathrm{keep}}(\cdot)$ as:

$$
=\sum_{(\sigma, \tau) \in \operatorname{conf}_{n}^{\text {keep }}(j, S, T, M)}^{\operatorname{Freq}_{n}^{\text {keep }}(j, S, T, M)=} p(\tau \mid \sigma) .
$$

Features for word deletions based on word alignments of partial matches $\left(A \operatorname{lig} n_{n}^{\text {keep }}\right)$ The third set of features takes advantage of partial matches, that is, of sub-segment pairs $(\sigma, \tau)$ in which $\tau$ does not appear as such in $T$. This set of features is defined as:

$$
=\sum_{\tau \in \text { segs_edop }_{n}(j, S, T, M, e)} \frac{\operatorname{Align}_{n}^{\text {keep }}(j, S, T, M, e)=}{\mid \frac{|\operatorname{LCS}(\tau, T)|}{|\tau|}}
$$

${ }^{3}$ Esplà-Gomis et al. (2015a) conclude that constraining only the length of $\tau$ leads to better results than constraining both $\sigma$ and $\tau$. where $\operatorname{LCS}(X, Y)$ returns the word-based longest common sub-sequence between segments $X$ and $Y$, and $\operatorname{segs} \_d o p_{n}(j, S, T, M, e)$ returns the set of sub-segments $\tau$ of length $n$ from $M$ that are a translation of a sub-segment $\sigma$ from $S$ and in which, after computing the LCS with $T$, the $j$-th word $t_{j}$ is assigned the edit operation $e:^{4}$

$$
\begin{aligned}
& \operatorname{segs\_ edop}_{n}(j, S, T, M, e)= \\
& =\left\{\left(\tau:(\sigma, \tau) \in M \wedge \sigma \in \operatorname{seg}_{*}(S)\right.\right. \\
& \left.\wedge|\tau|=n \wedge \operatorname{editop}\left(t_{j}, T, \tau\right)=e\right\}
\end{aligned}
$$

where editop $\left(t_{j}, T, \tau\right)$ returns the edit operation assigned to $t_{j}$ and $e$ is either delete or match. If $e=$ match the resulting set of features provides evidence in favour of keeping the word $t_{j}$ unedited, whereas when $e=$ delete it provides evidence in favour of removing it. Note that features $\operatorname{Align}_{n}^{\text {keep }}(\cdot)$ are the only ones to provide explicit evidence that a word should be deleted.

The three sets of features described so far, $\operatorname{Keep}_{n}(\cdot)$, Freq $_{n}^{\text {keep }}(\cdot)$, and Align keep $(\cdot)$, are computed for $t_{j}$ for all the values of sub-segment length $n \in[1, L]$. Features $\operatorname{Keep}_{n}(\cdot)$ and Freq $_{n}^{\text {keep }}(\cdot)$ are computed by querying the collection of subsegment pairs $M$ in both directions (SL-TL and TL-SL). Computing $\operatorname{Align}_{n}^{\text {keep }}(\cdot)$ only queries $M$ in one direction (SL-TL) but is computed twice: once for the edit operation match, and once for the edit operation delete.

\subsection{Features for insertion positions}

In this section, we describe three sets of features -based on those described in section 3.1 for word deletions - designed to detect insertion positions. The main difference between them is that the former apply to words, while the latter apply to gaps; we will call $\gamma_{j}$ the gap after word $t_{j}{ }^{5}$

Features for insertion positions based on subsegment pair occurrences (NoInsert) The first set of features, $\operatorname{NoInsert}_{n}(\cdot)$, based on the $\operatorname{Keep}_{n}(\cdot)$ features for word deletions, is defined as follows:

$$
\begin{array}{r}
\operatorname{NoInsert}_{n}(j, S, T, M)= \\
\frac{\left|\left\{\tau:(\sigma, \tau) \in \operatorname{conf}_{n}^{\text {noins }}(j, S, T, M)\right\}\right|}{\left|\left\{\tau: \tau \in \operatorname{seg}_{n}(T) \wedge[j, j+1] \subseteq \operatorname{span}(\tau, T)\right\}\right|}
\end{array}
$$

\footnotetext{
${ }^{4}$ Note that the sequence of edit operations needed to transform $X$ in $Y$ is a by-product of computing $\operatorname{LCS}(X, Y)$; these operations are insert, delete or match (when the corresponding word does not need to be edited).

${ }^{5}$ Note that the index of the first word in $T$ is 1 , and gap $\gamma_{0}$ corresponds to the space before the first word in $T$.
} 
where function $\operatorname{conf}_{n}^{\text {noins }}(j, S, T, M)$ returns the collection of sub-segment pairs $(\sigma, \tau)$ covering a given gap $\gamma_{j}$, and is defined as:

$$
\begin{array}{r}
\operatorname{conf}_{n}^{\text {noins }}(j, S, T, M)= \\
\left\{(\sigma, \tau) \in \operatorname{match}_{n}(M, S, T):\right. \\
[j, j+1] \subseteq \operatorname{span}(\tau, T)\}
\end{array}
$$

NoInsert $_{n}(\cdot)$ accounts for the number of times that the translation of sub-segment $\sigma$ from $S$ makes it possible to obtain a sub-segment $\tau$ that covers the gap $\gamma_{j}$, that is, a $\tau$ that covers both $t_{j}$ and $t_{j+1}$. If a word is missing in gap $\gamma_{j}$, one would expect to find fewer sub-segments $\tau$ that cover this gap, therefore obtaining low values for $\operatorname{NoInsert}_{n}(\cdot)$, while if there are no words missing in $\gamma_{j}$, one would expect more sub-segments $\tau$ to cover the gap, therefore obtaining values of $\operatorname{NoInsert}_{n}(\cdot)$ closer to 1 . In order to be able to identify insertion positions before the first word or after the last word, we use imaginary sentence boundary words $t_{0}$ and $t_{|T|+1}$, which can also be matched, ${ }^{6}$ thus allowing us to obtain evidence for gaps $\gamma_{0}$ and $\gamma_{|T|}$.

Features for insertion positions based on subsegment pair occurrences using translation frequency $\left(\right.$ Freq $_{n}^{\text {noins }}$ ) Analogously to Freq ${ }_{n}^{\text {keep }}(\cdot)$ above, we define the feature set Freq $_{n}^{\text {noins }}(\cdot)$, now for gaps:

$$
=\sum_{(\sigma, \tau) \in \operatorname{conf}_{n}^{\text {noins }}(j, S, T, M)}^{\operatorname{Freq}_{n}^{\text {noins }}(j, S, T, M)=} p(\tau \mid \sigma)
$$

Features for insertion positions based on word alignments of partial matches $\left(\operatorname{Align}_{n}^{\text {noins }}\right) \quad \mathrm{Fi}$ nally, the set of features $\operatorname{Align}_{n}^{\mathrm{keep}}(\cdot)$ for word deletions can be easily repurposed to detect the need for insertions by setting the edit operation $e$ in eq. (1) to match and insert and redefining eq. (2) as

$$
\begin{aligned}
& \operatorname{segs\_ } \_d o p_{n}(j, S, T, M, e)=\{(\tau:(\sigma, \tau) \in M \\
& \wedge|\tau|=n \\
& \left.\wedge \operatorname{editop}\left(t_{j}, \tau, T\right)=e\right\}
\end{aligned}
$$

where the LCS is computed between $\tau$ and $T$, rather than the other way round. ${ }^{7}$ We shall refer to this last set of features for insertion positions as $\operatorname{Align}_{n}^{\text {noins }}(\cdot)$.

\footnotetext{
${ }^{6}$ These boundary words are annotated in $M$ when this resource is built.

${ }^{7}$ It is worth noting that $\operatorname{LCS}(X, Y)=\operatorname{LCS}(Y, X)$, but the sequences of edit operations obtained as a by-product are different in each case.
}

The sets of features for insertion positions, $\operatorname{NoInsert}_{n}(\cdot)$, Freq $_{n}^{\text {noins }}(\cdot)$ and $\operatorname{Align}_{n}^{\text {noins }}(\cdot)$, are computed for gap $\gamma_{j}$ for all the values of subsegment length $n \in[2, L]$. As in the case of the feature sets employed to detect deletions, the first two sets are computed by querying the set of subsegment pairs $M$ via the SL or via the TL, while the latter can only be computed by querying $M$ via the SL for the edit operations insert and match.

\subsection{Neural network architecture and training}

We use a two-hidden-layer feed-forward NN to jointly predict the labels $(O K$ or $B A D)$ for word $t_{j}$ and gap $\gamma_{i}$, using features computed at word positions $t_{i-C}, t_{i-C+1}, \ldots, t_{i-1}, t_{i}, t_{i+1}, \ldots, t_{i+C-1}$, $t_{i+C}$ and at gaps $\gamma_{i-C}, \gamma_{i-C+1}, \ldots, \gamma_{i-1}, \gamma_{i}$, $\gamma_{i+1}, \ldots, \gamma_{i+C-1}, \gamma_{i+C}$, where $C$ represents the amount of left and right context around the word and gap being predicted.

The NN architecture has a modular first layer with ReLU activation functions, in which the feature vectors for each word and gap, with $F$ and $G$ features respectively, are encoded into intermediate vector representations ("embedding") of the same size; word features are augmented with the baseline features provided by the organizers. The weights for this first layer are the same for all words and for all gaps (parameters are tied). A second layer of ReLU units combines these representations into a single representation of the same length $(2 C+1)(F+G)$. Finally, two sigmoid neurons in the output indicate, respectively, if word $t_{i}$ has to be tagged as $B A D$, or if gap $\gamma_{i}$ should be labelled as $B A D$. Preliminary experiments confirmed that predicting word and gap labels with the same NN lead to better results than using two independent NNs.

The output of each of the sigmoid output units is additionally independently thresholded (Lipton et al., 2014) using a line search to establish thresholds that optimize the product of the $F_{1}$ score for $O K$ and $B A D$ categories on the development sets. This is done since the product of the $F_{1}$ scores is the main metric of comparison of the shared task, but it cannot be directly used as the objective function of the training as it is not differentiable.

Training was carried out using the Adam stochastic gradient descent algorithm to optimize crossentropy. A dropout regularization of $20 \%$ was applied on each hidden layer. Training was stopped when results on the development set did not improve for 10 epochs. In addition, each network was trained 10 times with different uniform initializa- 
tions (He et al., 2015), choosing the parameter set performing best on the development set.

Preliminary experiments have led us to choose a value $C=3$ for the number of words and gaps both to the left and to the right of the word and gap for which a prediction is being made; smaller values such a $C=1$ gave, however, a very similar performance.

\section{Experimental setting}

\subsection{Datasets provided by the organizers}

Six datasets were provided for the shared task on MT QE at WMT 2018 (Specia et al., 2018), covering four language pairs -English-German (ENDE), German-English (DE-EN), English-Latvian (EN-LV), and English-Czech (EN-CS) - and two MT systems — statistical MT (SMT) and neural MT (NMT). Each dataset is split into training, development and test sets. From the data provided by the organizers of the shared task, the approach in this paper used:

1. set of segments $S$ in source language,

2. set of translations $T$ of the SL segment produced by an MT system,

3. word-level MT QE gold predictions for each word and gap in each translation $T$, and

4. baseline features ${ }^{8}$ for word-level MT QE.

Regarding the baseline features, the organizers provided 28 features per word in the dataset, from which we only used the 14 numeric features plus the part-of-speech category (one-hot encoded). This was done for the sake of simplicity of our architecture. It is worth mentioning that no valid baseline features were provided for the EN-LV datasets. In addition, the large number of part-ofspeech categories in the EN-CS dataset led us to discard this feature in this case. As a result, 121 baseline features were obtained for EN-DE (SMT), 122 for EN-DE (NMT), 123 for DE-EN (SMT), 14 for EN-CS (SMT), and 0 for EN-LV (SMT) and EN-LV (NMT).

\subsection{External bilingual resources}

As described above, our approach uses ready-made, publicly available phrase tables as bilingual resources. In particular, we have used the cleaned phrase tables available on June 6, 2018 in OPUS for

\footnotetext{
${ }^{8}$ https: / / www.quest.dcs.shef.ac.uk/ quest_files/features_blackbox_baseline_ 17
}

the language pairs involved. These phrase tables were built on a corpus of about 82 million pairs of sentences for DE-EN, 7 million for EN-LV, and 61 million for EN-CS. Phrase tables were available only for one translation direction and some of them had to be inverted (for example, in the case of EN-DE or EN-CS).

\section{Results}

This section describes the results obtained by the UAlacant system in the MT QE shared task at WMT 2018 (Specia et al., 2018), which are reported in Table 1. Our team participated in two sub-tasks: sentence-level MT QE (task 1) and wordlevel MT QE (task 2). For sentence-level MT QE we computed the number of word-level operations predicted by our word-level MT QE approach and normalized it by the length of each segment $T$, in order to obtain a metric similar to TER. The words tagged as $B A D$ followed by gaps tagged as $B A D$ were counted as replacements, the rest of words tagged as $B A D$ were counted as deletions, and the rest of gaps tagged as $B A D$ were counted as oneword insertions. ${ }^{9}$ This metric was used to participate both in the scoring and ranking sub-tasks.

Columns 2 to 5 of Table 1 show the results obtained for task 1 in terms of the Pearson's correlation $r$ between predictions and actual HTER, mean average error (MAE), and root mean squared error (RMSE), as well as Sperman's correlation $\rho$ for ranking.

Columns 6 to 11 show the results for task 2 in terms of $F_{1}$ score both for categories $O K$ and $B A D,{ }^{10}$ together with the product of both $F_{1}$ scores, which is the main metric of comparison of the task. The first three columns contain the results for the sub-task of labelling words while the last three columns 9 to 11 contain the results for the sub-task of labelling gaps.

As can be seen, the best results were obtained for the language pair DE-EN (SMT). Surprisingly, the results obtained for EN-LV (NMT) were also specially high for word-level and sentence-level MT QE. These results for the latter language pair are unexpected for two reasons: first, because no baseline features were available for word-level MT

\footnotetext{
${ }^{9}$ Note that this approach is rather limited, as it ignores block shifts and the number of words to be inserted in a gap, which are basic operations to compute the actual TER value.

${ }^{10}$ For word deletion identification, a word marked as $B A D$ means that the word needs to be deleted, while in the case of insertion position identification, if a gap is marked as $B A D$ it means that one or more words need to be inserted there.
} 


\begin{tabular}{|l|rrr|r|rrr|rrr|}
\hline \multirow{2}{*}{ Dataset } & \multicolumn{4}{|c|}{ sentence-level } & \multicolumn{3}{c|}{ word-level (words) } & \multicolumn{3}{c|}{ word-level (gaps) } \\
\cline { 2 - 11 } & $r$ & MAE & RMSE & $\rho$ & $F_{\text {BAD }}$ & $F_{\text {OK }}$ & $F_{\text {MULTI }}$ & $F_{\text {BAD }}$ & $F_{\text {OK }}$ & $F_{\text {MULTI }}$ \\
\hline EN-DE SMT & 0.45 & 0.15 & 0.19 & 0.44 & 0.35 & 0.81 & 0.29 & 0.33 & 0.96 & 0.32 \\
EN-DE NMT & 0.35 & 0.14 & 0.20 & 0.41 & 0.22 & 0.86 & 0.19 & 0.12 & 0.98 & 0.12 \\
DE-EN SMT & 0.63 & 0.12 & 0.17 & 0.60 & 0.43 & 0.87 & 0.37 & 0.33 & 0.97 & 0.32 \\
EN-LV SMT & 0.36 & 0.20 & 0.26 & 0.34 & 0.27 & 0.82 & 0.22 & 0.15 & 0.94 & 0.14 \\
EN-LV NMT & 0.56 & 0.17 & 0.22 & 0.55 & 0.44 & 0.80 & 0.36 & 0.17 & 0.95 & 0.16 \\
EN-CS SMT & 0.43 & 0.18 & 0.23 & 0.46 & 0.42 & 0.75 & 0.31 & 0.15 & 0.95 & 0.15 \\
\hline
\end{tabular}

Table 1: Results for sentence-level MT QE (columns 2-5) in terms of the Pearson's correlation $r$, MAE, RMSE, and Sperman's correlation $\rho$ (for ranking). Results for the task of word labelling (columns 6-8) and gap labelling (columns 9-11) in terms of the $F_{1}$ score for class $B A D\left(F_{\mathrm{BAD}}\right)$, the $F_{1}$ score for class $O K\left(F_{\mathrm{OK}}\right)$ and the product of both ( $\left.F_{\text {MULTI }}\right)$.

QE task for this language pair, and second, because the size of the parallel corpora from which phrase tables for this language pair were extracted were an order of magnitude smaller. One may think that the coverage of machine translation by the phrase tables could have an impact on these results. To confirm this, we checked the fraction of words in each test set that were not covered by any subsegment pair $(\sigma, \tau)$. This fraction ranges from $15 \%$ to $4 \%$ depending on the test set, and has the lowest value for EN-LV (NMT); however, it is not clear that a higher coverage always leads to a better performance as one of the datasets with a better coverage was EN-LV (SMT) (5\%) which, in fact, obtained the worst results in our experiments.

It is worth noting that, when looking at the results obtained by other participants, the differences in performance between the different datasets seems to be rather constant, showing, for example, a drop in performance for EN-DE (NMT) and ENLV (SMT); this lead us to think that the test set might be more difficult in these cases. One thing that we could confirm is that, for these two datasets, the ratio of $O K / B A D$ samples for word-level MT $\mathrm{QE}$ is lower, which may make the classification task more difficult.

In comparison with the rest of systems participating in this task, UAlacant was the best-performing one in the sub-task of labelling gaps for 3 out of the 6 datasets provided (DE-EN SMT, EN-LV SMT, and EN-LV NMT). Results obtained for the subtask of labelling words were poorer and usually in the lower part of the classification. However, the sentence-level MT QE submissions, which build on the labels predicted for words and gaps by the word-level MT QE system, performed substantially better and outperformed the baseline for all the datasets but EN-DE (NMT) and, for EN-LV
(NMT), it even ranked third.

As said above, one of the main advantages of this approach is that it can be trained with limited computational resources. In our case, we trained our systems on a AMD Opteron(tm) Processor 6128 CPU with 16 cores and, for the largest set of features (dataset DE-EN SMT), training took 2,5 hours, about 4 minutes per epoch. ${ }^{11}$

\section{Concluding remarks}

We have presented a simple MT word-level QE method that matches the content of publicly available statistical MT phrase pairs to the source segment $S$ and its machine translation $T$ to produce a number of features at each word and gap. To predict if the current word has to be deleted or if words have to be inserted in the current gap, the features for the current word and gap and $C$ words and gaps to the left and to the right are processed by a twohidden-layer feed-forward NN. When compared with other participants in the WMT 2018 shared task, our system ranks first in labelling gaps for 3 of the 6 language pairs, but does not perform too well in labelling words. We also used word-level estimations to approximate TER. We participated with this approximation in the sentence-level MT QE sub-task obtaining a reasonable performance ranking, for almost all datasets, above the baseline.

One of the main advantages of the work presented here is that it does not require huge computational resources, and it can be trained even on a CPU in a reasonable time.

\section{Acknowledgments}

Work funded by the Spanish Government through the EFFORTUNE project (project number TIN2015-69632-R).

\footnotetext{
${ }^{11}$ Total training time corresponds to 35 epochs.
} 


\section{References}

Ergun Biçici. 2013. Referential translation machines for quality estimation. In Proceedings of the 8th Workshop on Statistical Machine Translation, pages 343-351, Sofia, Bulgaria.

Frédéric Blain, Carolina Scarton, and Lucia Specia. 2017. Bilexical embeddings for quality estimation. In Proceedings of the Second Conference on Machine Translation, pages 545-550.

J. Blatz, E. Fitzgerald, G. Foster, S. Gandrabur, C. Goutte, A. Kulesza, A. Sanchis, and N. Ueffing. 2004. Confidence estimation for machine translation. In Proceedings of the 20th International Conference on Computational Linguistics, COLING '04, pages 315-321, Geneva, Switzerland.

Ondrej Bojar, Christian Buck, Christian Federmann, Barry Haddow, Philipp Koehn, Johannes Leveling, Christof Monz, Pavel Pecina, Matt Post, Herve SaintAmand, Radu Soricut, Lucia Specia, and Aleš Tamchyna. 2014. Findings of the 2014 Workshop on Statistical Machine Translation. In Proceedings of the Ninth Workshop on Statistical Machine Translation, pages 12-58, Baltimore, MD, USA.

Ondřej Bojar, Rajen Chatterjee, Christian Federmann, Yvette Graham, Barry Haddow, Shujian Huang, Matthias Huck, Philipp Koehn, Qun Liu, Varvara Logacheva, Christof Monz, Matteo Negri, Matt Post, Raphael Rubino, Lucia Specia, and Marco Turchi. 2017. Findings of the 2017 Conference on Machine Translation (WMT17). In Proceedings of the Second Conference on Machine Translation, Volume 2: Shared Task Papers, pages 169-214, Copenhagen, Denmark. Association for Computational Linguistics.

Ondřej Bojar, Rajen Chatterjee, Christian Federmann, Yvette Graham, Barry Haddow, Matthias Huck, Antonio Jimeno Yepes, Philipp Koehn, Varvara Logacheva, Christof Monz, Matteo Negri, Aurelie Neveol, Mariana Neves, Martin Popel, Matt Post, Raphael Rubino, Carolina Scarton, Lucia Specia, Marco Turchi, Karin Verspoor, and Marcos Zampieri. 2016. Findings of the 2016 Conference on Machine Translation. In Proceedings of the First Conference on Machine Translation, pages 131-198, Berlin, Germany.

Ondřej Bojar, Rajen Chatterjee, Christian Federmann, Barry Haddow, Matthias Huck, Chris Hokamp, Philipp Koehn, Varvara Logacheva, Christof Monz, Matteo Negri, Matt Post, Carolina Scarton, Lucia Specia, and Marco Turchi. 2015. Findings of the 2015 Workshop on Statistical Machine Translation. In Proceedings of the Tenth Workshop on Statistical Machine Translation, pages 1-46, Lisbon, Portugal.

Miquel Esplà-Gomis, Felipe Sánchez-Martínez, and Mikel L. Forcada. 2015a. UAlacant word-level machine translation quality estimation system at WMT
2015. In Proceedings of the Tenth Workshop on Statistical Machine Translation, pages 309-315, Lisbon, Portugal.

Miquel Esplà-Gomis, Felipe Sánchez-Martínez, and Mikel L. Forcada. 2015b. Using on-line available sources of bilingual information for word-level machine translation quality estimation. In Proceedings of the 18th Annual Conference of the European Association for Machine Translation, pages 19-26, Antalya, Turkey.

Miquel Esplà-Gomis, Felipe Sánchez-Martínez, and Mikel L. Forcada. 2016. UAlacant word-level and phrase-level machine translation quality estimation systems at WMT 2016. In Proceedings of the First Conference on Machine Translation: Volume 2, Shared Task Papers, volume 2, pages 782-786.

Simona Gandrabur and George Foster. 2003. Confidence estimation for translation prediction. In Proceedings of the 7th Conference on Natural Language Learning at HLT-NAACL 2003 - Volume 4, CONLL '03, pages 95-102, Edmonton, Canada.

Kaiming He, Xiangyu Zhang, Shaoqing Ren, and Jian Sun. 2015. Delving deep into rectifiers: Surpassing human-level performance on imagenet classification. In Proceedings of the 2015 IEEE International Conference on Computer Vision (ICCV), ICCV'15, pages 1026-1034, Washington, DC, USA.

Hyun Kim, Hun-Young Jung, Hongseok Kwon, JongHyeok Lee, and Seung-Hoon Na. 2017a. Predictorestimator: Neural quality estimation based on target word prediction for machine translation. ACM Transactions on Asian and Low-Resource Language Information Processing (TALLIP), 17(1):3.

Hyun Kim, Jong-Hyeok Lee, and Seung-Hoon Na. 2017b. Predictor-estimator using multilevel task learning with stack propagation for neural quality estimation. In Proceedings of the Second Conference on Machine Translation, pages 562-568.

Julia Kreutzer, Shigehiko Schamoni, and Stefan Riezler. 2015. Quality estimation from ScraTCH (QUETCH): Deep learning for word-level translation quality estimation. In Proceedings of the Tenth Workshop on Statistical Machine Translation, pages 316-322, Lisbon, Portugal. Association for Computational Linguistics.

Zachary C. Lipton, Charles Elkan, and Balakrishnan Naryanaswamy. 2014. Optimal thresholding of classifiers to maximize F1 measure. In Machine Learning and Knowledge Discovery in Databases, pages 225-239, Berlin, Heidelberg. Springer Berlin Heidelberg.

André F. T. Martins, Ramón Astudillo, Chris Hokamp, and Fabio Kepler. 2016. Unbabel's participation in the WMT16 word-level translation quality estimation shared task. In Proceedings of the First Conference on Machine Translation, pages 806-811, Berlin, Germany. 
André FT Martins, Marcin Junczys-Dowmunt, Fabio N Kepler, Ramón Astudillo, Chris Hokamp, and Roman Grundkiewicz. 2017. Pushing the limits of translation quality estimation. Transactions of the Association for Computational Linguistics, 5:205-218.

Lucia Specia, Frédéric Blain, Varvara Logacheva, Ramón F. Astudillo, and André Martins. 2018. Findings of the WMT 2018 Shared Task on Quality Estimation. In Proceedings of the Third Conference on Machine Translation, Volume 2: Shared Task Papers, Brussels, Belgium.

Nicola Ueffing and Hermann Ney. 2005. Application of word-level confidence measures in interactive statistical machine translation. In Proceedings of the 10th European Association for Machine Translation Conference "Practical applications of machine translation”, pages 262-270, Budapest, Hungary.

Nicola Ueffing and Hermann Ney. 2007. Word-level confidence estimation for machine translation. Computational Linguistics, 33(1):9-40. 